\title{
JD12
}

\section{Electronic Publishing: Now and the Future}

\author{
Chairperson: A. G. Hearn \\ Editor: P. B. Boyce
}

